Published at Cortex:

https://www.sciencedirect.com/science/article/pii/S0010945221001751

\title{
Authentic and Posed Emotional Vocalizations Trigger Distinct Facial Responses
}

César F. Lima ${ }^{\mathrm{a}, b^{*}}$, Patrícia Arriaga ${ }^{\mathrm{a}}$, Andrey Anikin ${ }^{\mathrm{c}, \mathrm{d}}$, Ana Rita Pires ${ }^{\mathrm{a}}$, Sofia Frade ${ }^{\mathrm{a}}$, Leonor Neves ${ }^{\mathrm{a}}$, and Sophie K. Scott ${ }^{\mathrm{b}}$

${ }^{a}$ Instituto Universitário de Lisboa (ISCTE-IUL), Lisboa, Portugal

${ }^{\mathrm{b}}$ Institute of Cognitive Neuroscience, University College London, London, UK

${ }^{c}$ Equipe de Neuro-Ethologie Sensorielle (ENES) / Centre de Recherche en Neurosciences de Lyon (CRNL), University of Lyon/Saint-Etienne, CNRS UMR5292, INSERM UMR_S 1028, Saint-Etienne, France

${ }^{\mathrm{d}}$ Division of Cognitive Science, Lund University, Lund, Sweden

${ }^{*}$ Corresponding author

Cesar F. Lima Dhttps://orcid.org/0000-0003-3058-7204

Correspondence concerning this article should be addressed to César F. Lima, Iscte Instituto Universitário de Lisboa, Avenida das Forças Armadas, 1649-026 Lisboa, Portugal. E-mail: cesar.lima@iscte-iul.pt 


\begin{abstract}
The ability to recognize the emotions of others is a crucial skill. In the visual modality, sensorimotor mechanisms provide an important route for emotion recognition. Perceiving facial expressions often evokes activity in facial muscles and in motor and somatosensory systems, and this activity relates to performance in emotion tasks. It remains unclear whether and how similar mechanisms extend to audition. Here we examined facial electromyographic and electrodermal responses to nonverbal vocalizations that varied in emotional authenticity. Participants $(N=100)$ passively listened to laughs and cries that could reflect an authentic or a posed emotion. Bayesian mixed models indicated that listening to laughter evoked stronger facial responses than listening to crying. These responses were sensitive to emotional authenticity. Authentic laughs evoked more activity than posed laughs in the zygomaticus and orbicularis, muscles typically associated with positive affect. We also found that activity in the orbicularis and corrugator related to subjective evaluations in a subsequent authenticity perception task. Stronger responses in the orbicularis predicted higher perceived laughter authenticity. Stronger responses in the corrugator, a muscle associated with negative affect, predicted lower perceived laughter authenticity. Moreover, authentic laughs elicited stronger skin conductance responses than posed laughs. This arousal effect did not predict task performance, however. For crying, physiological responses were not associated with authenticity judgments. Altogether, these findings indicate that emotional authenticity affects peripheral nervous system responses to vocalizations. They also point to a role of sensorimotor mechanisms in the evaluation of authenticity in the auditory modality.
\end{abstract} Keywords: electrodermal activity; facial EMG; emotional authenticity; laughter; voice 


\section{Introduction}

The ability to perceive the emotional expressions of others is crucial for social behavior. Simulation models posit that sensorimotor mechanisms play an important role for emotion recognition. When we see a smiling face, for example, activating the processes involved in producing and experiencing that expression would provide a basis for inferring the other's state (e.g., Keysers \& Gazzola, 2009; Niedenthal et al., 2010; Wood et al., 2016). Supporting a role for sensorimotor mechanisms, neuroimaging, lesion, and stimulation studies document the involvement of motor and somatosensory systems during facial emotion perception, overlapping with the systems that control the production of facial movements (Adolphs et al., 2000; Hennenlotter et al., 2005; Korb et al., 2015; Pourtois et al., 2004). Facial electromyography (EMG) research indicates that such sensorimotor activity often results in facial muscle responses in the perceiver, called facial mimicry (Argaud et al., 2016; Fujimura et al., 2010; Hess \& Blairy, 2001; Korb et al., 2010). Facial mimicry can occur spontaneously, even when the task does not require emotional evaluations (e.g., Geangu et al., 2016; Isomura \& Nakano, 2016; Krumhuber et al., 2014). Mimicry can also relate to how facial expressions are subjectively perceived in terms of specific emotions (Künecke et al., 2014), authenticity (Korb et al., 2014), valence (Hyniewska \& Sato, 2015), and intensity (Lobmaier \& Fischer, 2015). This association is observed in correlational as well as in experimental studies, in which suppressing mimicry can lead to impaired emotion processing (Borgomaneri et al., 2020; Maringer et al., 2011; Oberman et al., 2007; Rychlowska et al., 2014; but see Hess, 2021).

Central to the understanding of these findings is the question of whether sensorimotor mechanisms are specifically involved in facial emotion processing, or provide a general route for emotion processing across modalities (e.g., audition). In the current study, we examined facial muscle responses to emotional vocalizations. The human voice is a primary channel for 
emotional communication (e.g., Cowen et al., 2019), but vocal expressions have been commonly overlooked in the sensorimotor simulation literature. The literature on vocal emotions, on the other hand, has mostly focused on subcortical, auditory and cognitive contributions to perception, not on sensorimotor mechanisms (Frühholz et al., 2016; Grandjean, 2020; Schirmer \& Kotz, 2006). Moreover, facial EMG studies have been crucial for documenting sensorimotor processes in the visual modality, and similar studies for vocal emotions are rare (Arias et al., 2018; Hietanen et al., 1998; Magnée et al., 2007). The literature on peripheral nervous system responses to vocal emotions is in fact scant.

Like facial expressions, vocalizations such as laughter and crying provide a window into the emotions and intentions of others. They are rapidly detected (Sauter \& Eimer, 2010), accurately recognized even under cognitive load (Lima et al., 2014; Lima et al., 2019; Sauter et al., 2010a), and their meaning is shared across cultures (Sauter et al., 2010b). Most studies focus on how vocalizations communicate specific emotions, but an emerging body of work is exploring how variability within the same vocalization can reflect distinct socio-emotional processes. Research on laughter, a pervasive expression of emotion, indicates a distinction between spontaneous and voluntary laughs (Bryant \& Aktipis, 2014; Bryant et al., 2018; Gervais \& Wilson, 2005; McKeown et al., 2015; Scott et al., 2014). Spontaneous laughs reflect a genuinely felt emotion, are less controlled, and are typically reactive to outside events. Voluntary laughs are part of more deliberate communicative acts. They are flexibly used to express polite agreement, appreciation, or to deceive others. Being able to detect the authenticity of emotional expressions is an important skill, and listeners can distinguish between authentic and voluntary laughs (e.g., Bryant et al., 2018; Lavan et al., 2016; Neves et al., 2018). This sensitivity is also seen at the cortical level. In an fMRI study of passive listening to laughter, McGettigan et al. (2015) found that spontaneous laughs evoke more activity than voluntary laughs in superior temporal gyri, and voluntary laughs evoke more 
activity in medial prefrontal and cingulate cortices. This activity suggests an engagement of mentalizing processes when laughs are not authentic. Studies on vocalizations beyond laughter are scant, but there is evidence that listeners can tell authentic from voluntary expressions for emotions such as crying (Anikin \& Lima, 2018).

Sensorimotor mechanisms might contribute more to emotion processing when the context is challenging; when the expression is subtle or ambiguous, or the inference to be made is complex (Lima et al., 2016b; Wood et al., 2016). Authenticity detection in vocalizations requires a complex judgment of intentionality based on subtle acoustic cues. It therefore offers an ideal task to ask whether facial responses play a role for auditory emotion processing. Such a role can be hypothesized from previous studies. In line with findings for faces, motor system activation, in primary motor, premotor, and supplementary motor sites, is seen for the perception of emotional vocalizations (Bestelmeyer et al., 2014; Lima et al., 2015; Lima et al., 2016b; McGettigan et al., 2015; O’Nions et al., 2017). This activation is particularly seen for positive vocalizations like laughter, possibly reflecting the contagiousness of these expressions (Warren et al., 2006). Suppression of the somatosensory and premotor cortices activity can disrupt emotion recognition in vocalizations (Banissy et al., 2010), and children with stronger connectivity between the inferior frontal and motor regions display better vocal emotion recognition performance (Correia et al., 2019). Concerning laughter authenticity, there is evidence that sensorimotor activity can relate to behavioral outcomes. Stronger responses in the motor system during listening to authentic and posed laughs correlated with improved authenticity detection (McGettigan et al., 2015). We have also found that the propensity to engage in sensorimotor activity, as a trait evaluated via self-report, is associated with improved laughter authenticity detection (Neves et al., 2018). Moreover, facial responses to vocalizations would represent a case of cross-modal influence, not a strict 'imitation' of the stimulus, but everyday emotional vocalizations are 
indissociable from facial activity (e.g., Hawk et al., 2012). They could not be mimicked without facial movements.

Here we addressed two novel questions. First, we aimed to conduct the first investigation of peripheral nervous system correlates of emotional authenticity in vocalizations. We asked whether facial muscles respond differently when we listen to authentic compared to posed vocal expressions. The stimuli included laughs and cries. We examined three muscles: zygomaticus (zygomaticus major, pulls the lip corners up), orbicularis (orbicularis oculi, produces wrinkles around the eye socket), and corrugator (corrugator supercilii, lowers and furrows the eyebrows; see Figure 1). The corrugator is typically associated with negative emotions, such as anger and sadness, while the zygomaticus and orbicularis are involved in smiling and are associated with positive emotions (Dimberg et al., 2000; Dimberg et al., 2002; Grainger et al., 2019). The orbicularis is often considered a mark of authentic affect in so-called Duchenne smiles (e.g., Korb et al., 2014). Our second aim was to ask whether facial responses to vocalizations are associated with subjective evaluations of emotional authenticity. Related to this aim, we also examined electrodermal responses, which index arousal mediated by autonomic nervous system activity. Authentic vocalizations are often higher in perceived arousal (e.g., Bryant et al., 2018; Lavan et al., 2016). We wanted to determine whether this is manifested in physiological arousal too, and whether the potential associations between facial activity and perceived authenticity is also seen for the more unspecific arousal response.

Participants completed an implicit emotion processing task, during which facial and electrodermal responses were recorded. For most vocalizations, they passively listened to the sounds, without providing any motor response. For a minority of them, the vigilance trials, they were prompted to identify the sex of the speaker. By directing participants' attention to the sex of the speaker we ensured that they remained vigilant, and that they were unlikely to 
detect that our focus was on authenticity. In fact, they were not informed that the stimuli varied in authenticity. After the implicit task, participants listened to the vocalizations again and made explicit authenticity evaluations.

We expected facial muscle responses to be stronger for laughter than for crying, based on evidence that laughter is particularly contagious (e.g., Scott et al., 2014), and that positive emotions preferentially engage sensorimotor systems (Warren et al., 2006). Facial responses should be seen in the zygomaticus and orbicularis for laughter, because of their association with positive emotions, and in the corrugator for crying, because of their association with negative emotions. If sensorimotor mechanisms play a role in vocal emotions, we could see distinct facial responses to authentic and posed vocalizations, and an association between these responses and authenticity judgments. These predictions were informed by evidence from laughter and visual smile perception. Sensorimotor cortical responses have been shown to relate to authenticity perception in laughter (McGettigan et al., 2015), and Duchenne smiles - typically associated with authentic feelings - have been shown to elicit stronger mimicry than non-Duchenne ones (Krumhuber et al., 2014; Surakka \& Hietanen, 1998). There is also a role of smile mimicry in authenticity perception (Korb et al., 2014; Maringer et al., 2011; Rychlowska et al., 2014). For electrodermal activity, we expected greater increases in arousal for authentic than for posed vocalizations, and explored associations with authenticity detection. Finally, participants completed self-report measures of emotional resonance, in an attempt to replicate the finding that the propensity to engage in sensorimotor activity predicts authenticity detection (Neves et al., 2018).

\section{Method}

\subsection{Participants}


One-hundred participants ( 67 female) were tested ${ }^{1}$. They were 24.2 years of age on average $(S D=5.1$, range $=18-40)$, and were recruited from research participant pools, or in response to advertisements on social media or on campus. According to self-reports, all had normal hearing, normal or corrected-to-normal vision, and no history of psychiatric or neurological disorders (these inclusion criteria were established at the recruitment stage). They were native Portuguese speakers, apart from two who had Portuguese as their second language.

Ethical approval for the study protocol was obtained from the local Ethics Committee, Iscte - University Institute of Lisbon (reference 23/2019). Written informed consent was collected from all participants, who were either offered a voucher or given partial course credit to take part.

\subsection{Materials}

\section{Vocal Stimuli}

The experimental stimuli consisted of 80 vocalizations, with 20 different vocalizations representing each of four conditions: posed laughter, authentic laughter, posed crying, and authentic crying (in each condition, 10 stimuli were produced by men and 10 by women). The vocalizations were generated in a sound-proof anechoic chamber at University College London by six speakers, three women and three men (aged 24 to 48 years).

Authentic laughter was elicited using an amusement induction situation in a social interactive setting. The speakers were shown video clips, which they identified beforehand as amusing and that would easily make them laugh. The experimenters knew the speakers well and interacted with them during the recording session to promote the naturalness and the social nature of the laughs, as they occur among friends in everyday social interactions. Authentic crying was also obtained via emotion induction: the speakers recalled upsetting

\footnotetext{
${ }^{1}$ This number is in line with a previous study conducted using similar stimuli and task (Neves et al., 2018)
} 
events and/or initially posed crying to encourage a transition into authentic crying, associated with felt sadness. During the debriefing, all speakers reported having experienced feelings of amusement and sadness during and after recording the corresponding expressions. For posed laughter and crying, the same speakers simulated the expressions in the absence of external stimulation, while trying to make the expression sound credible, following the same general procedure typically used for the recording of acted stimuli (e.g., Lima et al., 2013).

These stimuli have been used frequently in behavioral and neuroimaging studies (Lavan et al., 2015; Lavan et al., 2016; Lima et al., 2016a; O’Nions et al., 2017). The final set of 80 vocalizations was selected from a larger pool of stimuli based on a pilot perceptual validation study ( $N=26$ listeners, none of which took part in the main study). On a sevenpoint rating scale (1-7), authentic laughs were perceived as more authentic $(M=5.2 ; S D=$ $0.7)$ than posed laughs $(M=3.4 ; S D=0.5)$, and authentic cries as more authentic $(M=4.6$; $S D=0.4)$ than posed cries $(M=3.0 ; S D=0.8)$. The duration of the vocalizations was 2.469 ms on average $(S D=395)$, and it was relatively similar for posed and authentic expressions (2.394 $\mathrm{ms}$ and $2.545 \mathrm{~ms}$, respectively).

Sixteen additional laughter and crying vocalizations were included in the implicit task for vigilance trials, in which participants were prompted to identify the sex of the speaker (see Procedure, 2.3.). These consisted of a mix of authentic and posed stimuli and were produced by the same speakers as the experimental vocalizations.

\section{Emotional Contagion Scale (ECS)}

The ECS is a unidimensional 15-item questionnaire that assesses the propensity to resonate with others' emotions (Doherty, 1997; Portuguese version, Rueff-Lopes \& Caetano, 2012). It was used as a measure of trait levels of sensorimotor responses as in Neves et al. (2018). Scale items cover contagion for five emotions: love (e.g., I melt when the one I love holds me close), happiness (e.g., Being around happy people fills my mind with happy 
thoughts), sadness (e.g., I cry at sad movies), anger (e.g., It irritates me to be around angry people), and fear (e.g., I notice myself getting tense when I'm around people who are stressed $o u t$ ). Items are rated on a five-point Likert scale from 1 (never) to 5 (always). The ECS and its Portuguese translation have good psychometric properties, including high internal consistency, convergent and discriminant validity, and test-retest reliability (Doherty, 1997; Rueff-Lopes \& Caetano, 2012). Item scores are averaged to produce a total contagion score. Internal consistency values were good in the current dataset (Cronbach's $\alpha=.84$ ).

\section{Interpersonal Reactivity Index (IRI)}

The IRI is a 28 -item questionnaire that assesses individual differences in trait empathy (Davis, 1980, 1983; Portuguese version, Limpo et al. 2010). Scale items are grouped into four subscales, each of them corresponding to a different facet of empathy: perspective taking (e.g., I try to look at everybody's side of a disagreement before I make a decision), fantasy (e.g., I really get involved with the feelings of the characters in a novel), personal distress (e.g., In emergency situations, I feel apprehensive and ill-at-ease), and empathic concern (e.g., I am often quite touched by things that I see happen). Items are rated on a five-point Likert scale from 0 (does not describe me well) to 4 (describes me very well). The IRI and its Portuguese translation have appropriate psychometric properties (Davis, 1980, 1983; Limpo et al., 2010). The Portuguese IRI has only 24 items because four items were excluded to improve the adjustment of the data to the expected factorial structure of the scale (Limpo et al., 2010). Item scores are averaged to produce scores for each facet of empathy. Internal consistency values were acceptable-to-good in the current dataset (Cronbach's $\alpha=.75$ for perspective taking, $\alpha=.88$ for fantasy, and $\alpha=.81$ for personal distress, and $\alpha=.81$ for empathic concern). As in Neves et al. (2018), we focused our analyses on affective empathy, namely on the Empathic Concern subscale, which measures trait levels of affective reactions to others' emotions. Personal Distress also focuses on affective reactions, but is self-oriented. 


\subsection{Procedure}

Participants were tested individually, in a single experimental session lasting about 1.5 hours. They read and signed the consent form, and then the electrodermal and facial EMG sensors were attached. They were seated comfortably in an armchair about 65 centimeters away from a 23-inch LCD display (LG Flatron W236 3D), with their non-dominant arm and hand placed at resting position. They were asked to avoid moving their non-dominant hand, in which the electrodermal sensors were attached, and to use their dominant hand to fill the questionnaires and perform the tasks throughout the session.

The testing started with participants completing a background survey that asked for demographic information, and the ECS and IRI questionnaires. An initial psychophysiological baseline of three minutes was acquired, followed by the implicit task, during which psychophysiological responses to the emotional vocalizations were measured. E-Prime 2 (version 2.0.10.356; Psychology Software Tools Inc.) was used to present the vocalizations and collect behavioral responses. The sounds were played binaurally through high-quality headphones (Beyerdynamic DT 770 Pro, 80 Ohm), with the volume individually adjusted to a comfortable level. Participants were instructed to listen to each vocalization, and were told that for some of them (vigilance trials) they would be prompted to identify the sex of the speaker by pressing a prespecified key on the keyboard (1 for man, 2 for woman). Experimental $(n=80)$ and vigilance vocalizations $(n=16)$ were intermixed and presented in a random order for each participant. Each stimulus was presented only once. Vigilance trials were intended to ensure that participants remained attentive throughout the task, and that their attention was not focused on authenticity. Each trial was structured as follows. A fixation cross was presented for 1 second as a pre-stimulus baseline, followed by the auditory presentation of the stimulus for $\approx 3$ seconds with a blank screen. Then the blank screen remained for 5 seconds, after which the next trial started (experimental trials), or participants 
were prompted to identify the sex of the speaker by a question on the screen (was this vocalization produced by a man or a woman?; vigilance trials). Each experimental trial therefore lasted $\approx 9$ seconds. Physiological responses during vigilance trials were not analyzed.

The explicit task was completed after participants finished the implicit one. They were told that they would listen to the same vocalizations again, and informed that some of them reflected an authentic emotion, whereas others were posed (i.e., speakers were acting out the expressions, without feeling the emotion). They evaluated emotional authenticity on a seven-point Likert scale from 1 (posed) to 7 (authentic). Each trial was structured as follows. A fixation cross was presented for 1 second, followed by the auditory presentation of the stimulus for $\approx 3$ seconds with a blank screen. Then the response scale appeared on the screen until participants responded or for a maximum of 4 seconds, after which the next trial started. No feedback was provided and the task started with five practice trials.

Due to software malfunction, psychophysiological data from seven participants were not correctly recorded. These participants were therefore excluded from the analyses.

As part of a separate project, participants also wore attached electrocardiogram sensors, and their heart activity was monitored during the implicit task. These results will be reported in a separate publication.

\subsection{Psychophysiological Measurement}

Psychophysiological data were acquired via BIOPAC MP150 (Biopac Systems Inc.), using the EDA100C Electrodermal response and EMG100C Electromyogram amplifiers, and the recording software AcqKnowledge (version 4.1). Both facial EMG and electrodermal activity were recorded with a sampling rate of $1000 \mathrm{~Hz}$. Electrodermal activity was measured on the palmar surface of the middle phalanges of the index and middle fingers of the nondominant hand. A constant voltage of $0.5 \mathrm{~V}$ was maintained between two disposable $6 \mathrm{~mm}$ 
$\mathrm{Ag} / \mathrm{AgCl}$ snap electrodes filled with $\mathrm{NaCl}$ electrolyte paste gel. Facial data were measured at the left corrugator, zygomatic and orbicularis muscles, using six disposable $4 \mathrm{~mm} \mathrm{Ag} / \mathrm{AgCl}$ electrodes filled with conductive gel and placed according to common recommendations (Fridlund \& Cacioppo, 1986; Lapatki et al., 2003). The experimental setup is illustrated in Figure 1A and the effects of muscle activity on facial features in Figure 1B.

The resulting raw measurements were preprocessed offline using AcqKnowledge (versions 4.3 and 5). Based on visual inspection, the quality of the data was generally good, without significant interference induced by motion. Electrodermal data were first resampled to $62.5 \mathrm{~Hz}$, processed using a 62-sample median smoothing filter, and a finite impulse response (FIR) low-pass filter at $1 \mathrm{~Hz}$ with a Blackman (-61 dB) window (e.g., Braithwaite et al., 2013). For our analyses, we considered the phasic component of electrodermal activity, i.e., skin conductance responses (SCRs) for each vocalization. These responses were obtained using an in-built routine in AcqKnowledge, by implementing a $0.05 \mathrm{~Hz}$ high pass filter, a baseline estimation window width of $0.25 \mathrm{~s}$, a SCR threshold level at 0.02 microsiemens $(\mu \mathrm{S})$, and a rejection rate of $10 \%$. SCRs were calculated as the trough-to-peak amplitude difference (in $\mu \mathrm{S}$ ) of the largest deflection in the 1-4 s latency window after stimulus onset.

Facial data were preprocessed by first applying a FIR band-pass filter between 28 and $500 \mathrm{~Hz}$, with a Blackman (-61 dB) window. The raw data were transferred into EMG signals by calculating the root-mean-square (RMS) every 1000 samples. 

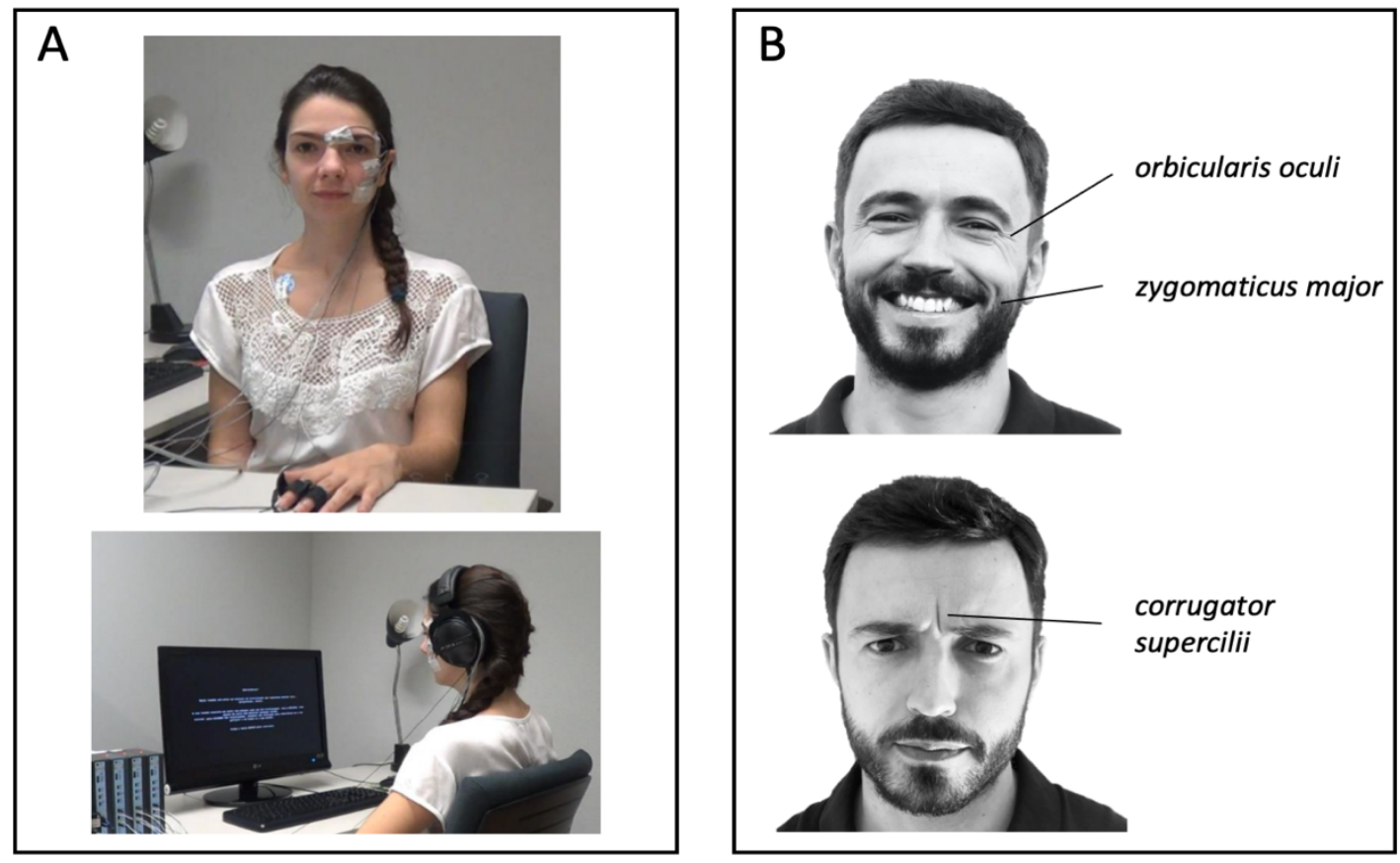

Figure 1. Illustration of the experimental setup (A) and of the effects of muscle activity on facial features (B). Used with permission from Camilla Silveira and César Lima.

\subsection{Data Analysis}

Statistical analyses were performed based on unaggregated data from individual trials, using Bayesian mixed models fitted with the help of the $\mathrm{R}$ package brms with default, mildly conservative priors (Bürkner, 2017). All results were summarized as the medians of posterior distributions and 95\% credible intervals (CI). When contrasting two conditions (e.g., authentic vs. posed vocalizations), the CI includes the most credible values for the difference given the data and the model, and if that does not include 0 we can infer that there is evidence in favor of an actual difference between conditions. The code used for data analysis, the full data set, and additional study materials, can be found here:

https://osf.io/nvb2u/?view_only=93cc2a9a741240f3adbe02c94cc9fdc8

No part of the study analyses was pre-registered prior to the research being conducted. However, we report how we determined our sample size, all data exclusions, all 
inclusion/exclusion criteria, whether inclusion/exclusion criteria were established prior to data analysis, all manipulations, and all measures in the study.

\section{Behavioral data and questionnaires}

According to recent guidelines (Bürkner, 2019), we modeled the 1-7 authenticity ratings with ordinal logistic regression. Because each stimulus was rated repeatedly, we included a random intercept per sound. To account for possible differences between participants, we also allowed the effects of authenticity and vocalization type (laughter or crying) to vary across subjects, so the main model of authenticity ratings was of the form: Auth_rating $\sim$ Authenticity * Vocalization $+($ Authenticity $*$ Vocalization $\mid$ Subject $)+(1 \mid$ Sound $)$ Demographic data (sex and age) and questionnaire results (ECS and IRI scores) were added to these models as covariates for analyses focused on these variables.

\section{$E M G$ data}

From the preprocessed data, EMG values for the three muscles (measured in microvolts, $\mu \mathrm{V}$ ) were downsampled to $2 \mathrm{~Hz}$ for statistical analyses. For each experimental trial, the time series were averaged across sequential $500 \mathrm{~ms}$ intervals, between 1 second before stimulus onset (pre-stimulus baseline), over 8 seconds after stimulus onset. Values were then log-transformed to remove the pronounced right skew in their distribution. To account for inter-individual differences in physiological activity, we subtracted the logtransformed pre-stimulus baseline from the log-transformed post-stimulus measurements (for an analogous approach, see e.g., Argaud et al., 2016; Korb et al., 2014; Krumhuber et al., 2014). This provides a difference score indicating changes in muscle activity in response to the vocalizations, but the magnitude of the response itself might also depend on the baseline level of activity. If a participant is already smiling before hearing a laugh, for example, changes in the zygomaticus and orbicularis can hardly be expected to be as strong as in trials in which the face is fully relaxed beforehand. We therefore modeled the activation of facial 
muscles relative to their state just before the stimulus was heard, after adjusting for how this pre-stimulus state compared to a neutral state before the experiment began (for a similar approach, Oliva \& Anikin, 2018):

$$
\text { Resp_adj } \sim \text { Authenticity *Vocalization + Base_adj }+(\text { Authenticity } * \text { Vocalization } \mid \text { Subject })+
$$
(1|Sound),

where

Adjusted response (Resp_adj) $=\log ($ response $/$ pre-trial baseline $)$

and

Adjusted baseline (Base_adj) $=\log ($ pre-trial baseline $/$ pre-experiment baseline)

The fitted values of adjusted response were then transformed into percent change

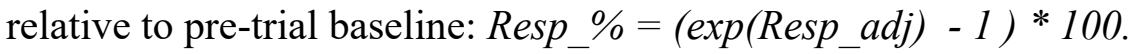

\section{Electrodermal data}

We focused on the amplitude of responses for vocalizations in which there was an SCR, and on the number of SCRs per condition. Amplitude values were log-transformed to remove skewness. Because SCRs and their amplitudes were defined as difference scores, no explicit correction for pre-trial baseline was necessary, but otherwise we followed the same modeling approach as for EMG data and again controlled for pre-experiment baseline.

\section{Results}

\subsection{Behavioral data}

The accuracy of sex discrimination during the implicit task was high $(M=92.5 \%$ per participant; $S D=9.6 \%$; range $=44-100$ ), indicating that participants remained attentive to the vocalizations while physiological responses were being recorded.

Regarding the explicit authenticity task, we evaluated inter-rater agreement by calculating the mean Pearson's correlation between individual responses and the overall authenticity ratings averaged across all participants. This correlation was $r=.70$ for laughter 
(intraclass correlation coefficient, ICC $=.40,95 \% \mathrm{CI}[.31, .53])$ and $r=.58$ for crying (ICC $=$ $.27,95 \%$ CI $[.19, .38])$, demonstrating moderate inter-rater agreement.

The average authenticity rating across all stimuli was 4.2 on the $1-7$ scale ( $95 \% \mathrm{CI}$ $[4.0,4.5])$, indicating a slight bias towards evaluating vocalizations as 0.25 points $(95 \% \mathrm{CI}$ $[0.04,0.46])$ more authentic than the 'neutral' rating of 4 . This bias was considerable for laughter (average fitted authenticity $=4.6,95 \% \mathrm{CI}[4.3,4.8])$, but not for crying $(3.9,95 \% \mathrm{CI}$ $[3.6,4.2])$. Regardless of their real or posed nature, laughs were perceived as 0.6 points more authentic than cries $(95 \%$ CI $[0.3,1.0])$.

As can be seen in Figure 2, participants perceived authentic laughs and cries as more authentic than their posed counterparts, with a difference of 2.2 points $(95 \% \mathrm{CI}[1.8,2.5])$. The magnitude of authenticity discrimination (i.e., difference between authentic and posed) was slightly greater for laughter $(2.4,95 \%$ CI $[1.9,2.8])$ than for crying $(1.9,95 \%$ CI $[1.4$, 2.4]), but in both cases participants could clearly tell authentic from posed expressions.

We found no evidence that the listeners' sex and age affected authenticity ratings. The difference in authenticity ratings provided by male vs. female listeners was only 0.01 points (95\% CI $[-0.3,0.3])$. Furthermore, adding Sex or Age with an interaction with Vocalization and Authenticity failed to improve the model fit as suggested by the LOO Information Criterion (LOOIC), which is a Bayesian approximation to leave-one-out cross-validation (Sex: difference in LOOIC $=-0.4, S E=1.7$; Age: difference $=-0.9, S E=1.3$ ). 


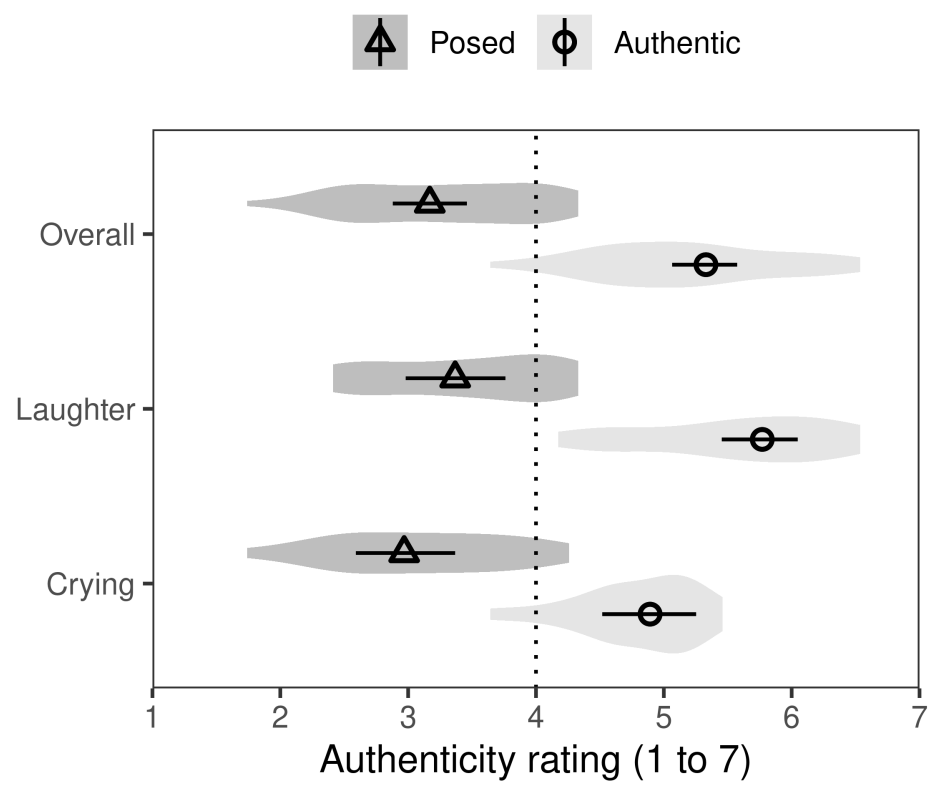

Figure 2. Authenticity ratings of authentic and posed laughter and crying. The points show fitted values from ordinal mixed models, with 95\% CIs. The violin plots show the distribution of observed ratings aggregated per sound. The dashed line indicates the neutral mid-point of the scale.

\subsection{Physiological data}

\section{Facial EMG}

Figure 3 shows responses in each of the three facial muscles, separately for authentic and posed laughs and cries. As predicted, responses were stronger for laughter compared to crying. During listening to laughter, there was a selective activation of the zygomaticus and orbicularis, with a fast onset and peak around $\sim 3.5 \mathrm{~s}$ after the beginning of the sound. Consistent with our hypothesis, this activation was higher for authentic compared to posed 
laughs. This effect was supported by analyses of average and peak levels of activity (see Figure 3B).

Looking at average activity, considering all time bins after sound onset, the difference between authentic and posed laughs in the orbicularis was $8.5 \%(95 \% \mathrm{CI}[4.5,12.8])$, and in the zygomaticus it was $6.2 \%(95 \% \mathrm{CI}[2.8,9.9])$. In the corrugator, by contrast, no difference was found, although the trend was in the opposite direction: lower activity for authentic vs. posed laughs, $-0.8 \%(95 \%$ CI $[-2.3,0.7])$. Listening to crying did not elicit responses credibly different from 0 in any of the three muscles (Figure 3). We also did not find differences between authentic and posed cries (orbicularis, difference $0.2 \%, 95 \%$ CI $[-2.8,3.2]$; zygomaticus, $0.7 \%, 95 \%$ CI $[-1.9,3.1]$; corrugator, $0.4 \%, 95 \%$ CI $[-0.9,1.7])$. Model fit was not improved by including an interaction between average facial muscles activity and the listener's sex (difference in LOOIC $=4.4$ in favor of the simpler model, $S E=3.9$ ) or age (difference $=6.9, S E=4.2$ ) as predictors of authenticity ratings.

For peak activity, the advantage of authentic over posed laughs in the orbicularis was $15.1 \%(95 \% \mathrm{CI}[7.6,23.2])$, and in the zygomaticus it was $12.9 \%(95 \% \mathrm{CI}[6.1,20.3])$. Again, no differences were found in the corrugator, $0.8 \%(95 \% \mathrm{CI}[-2.0,3.5])$. Listening to crying did not elicit peak responses different from 0 in any of the muscles, and we did not find any difference between authentic and posed cries (orbicularis, difference $0.1 \%, 95 \% \mathrm{CI}$ [-6.0, 5.8]; zygomaticus, $0.6 \%, 95 \%$ CI [-4.3, 5.8]; corrugator, $1.2 \%, 95 \%$ CI $[-1.6,4.0])$. 

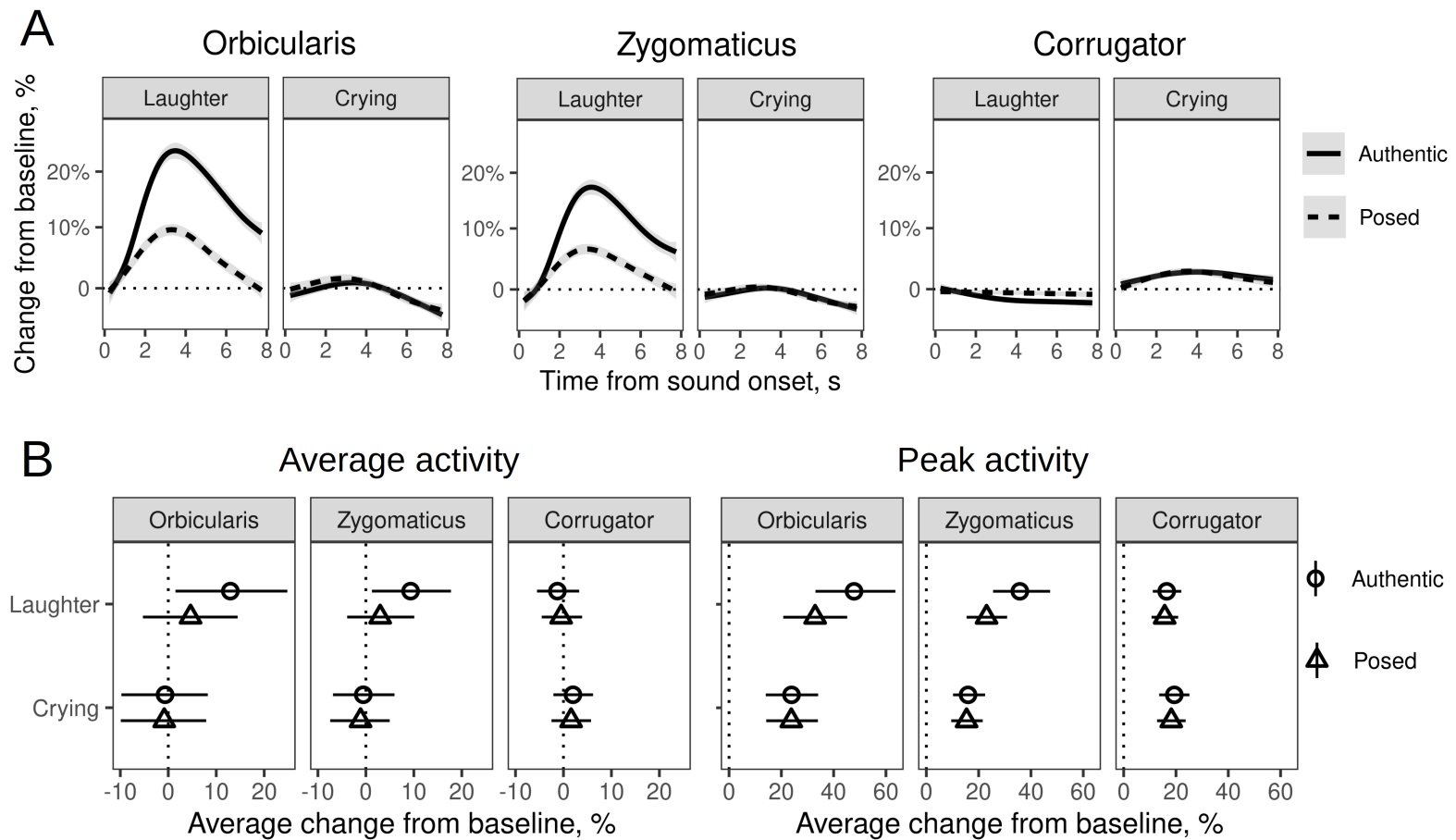

Figure 3. Facial activity in the orbicularis, zygomaticus and corrugator muscles during passive listening to authentic and posed laughter and crying. The graphs show percent change as compared to pre-trial baseline, as a time series smoothed with cubic regression splines (A), or as average and peak change considering all time bins after sound onset (B).

To examine whether facial responses related to authenticity evaluations, we first focused on how average activity predicted authenticity ratings. These effects are depicted in Figure 4. An increase in the activation of the orbicularis over the observed range of values predicted credibly higher authenticity ratings for authentic laughs (1.8 points higher ratings, $95 \%$ CI $[0.6,2.8])$, as well as for posed laughs $(1.9,95 \%$ CI $[0.3,3.4])$. A similar association could not be identified for zygomaticus activity (authentic laughs, difference -0.1 points, 95\% CI $[-1.5,1.3]$; posed laughs, difference -1.2 points, 95\% CI $[-3.0,0.8])$.

For the corrugator, an increase over the observed range of values predicted lower authenticity ratings, even though the effect was statistically uncertain (authentic laughs, 
difference -1.7 points, $95 \%$ CI $[-3.2,0.2]$; posed laughs, difference -2.3 points, $95 \%$ CI [-4.3, 0.3]). Thus, stronger responses in the orbicularis, a muscle typically associated with positive/authentic affect, were associated with greater perceived laughter authenticity (regardless of whether laughs were authentic or posed). Stronger responses in the corrugator, a muscle often associated with negative affect, were associated with slightly lower perceived laughter authenticity. This pattern is similar in the analyses focusing on peak rather than average muscle activity, and no associations were found between muscle activity and authenticity detection in crying (Figure 4).

\section{$\phi$ Authentic 4 Posed}

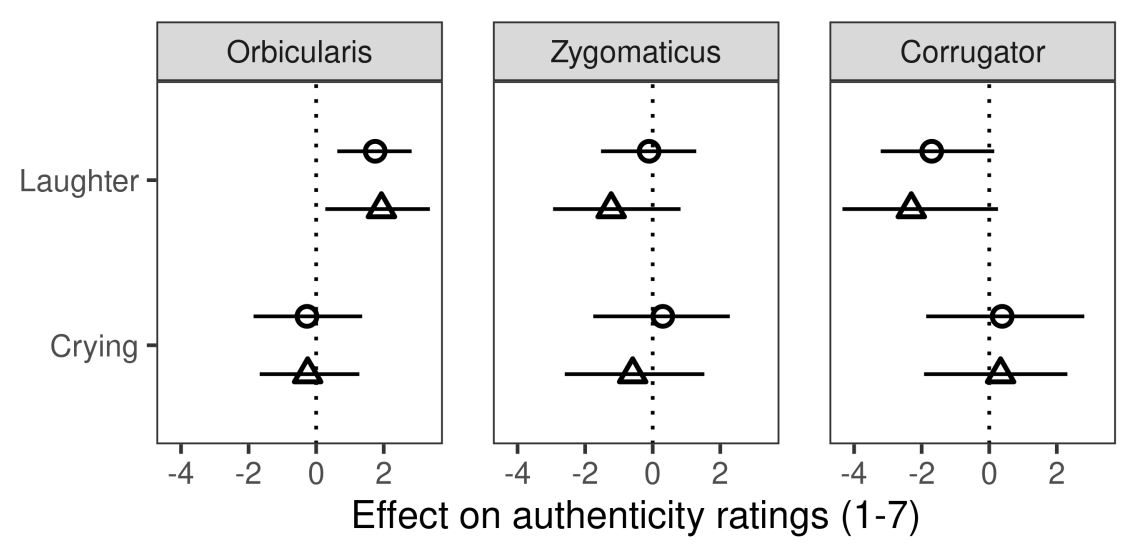

Figure 4. The effect of average facial muscles activity on authenticity ratings. Shown: the predicted effect of increasing average facial responses over their observed range of values.

These analyses reveal associations between the magnitude of facial responses and authenticity judgments, but this does not necessarily mean better performance (e.g., stronger orbicularis activity, when in response to posed laughs, could create an 'illusion' of authenticity). Better performance would require more selective facial responses across laughter types. In participant-based follow-up analyses, we asked whether participants 
showing more differentiated facial responses across laughter types (i.e., a larger difference score in activity for authentic laughs - activity for posed laughs) also showed more differentiated authenticity discrimination (i.e., a large difference score in ratings for authentic laughs - ratings for posed laughs). This was not found for the corrugator, -0.8 (95\% CI [-2.4, 0.8]). But for the zygomaticus and orbicularis, the muscles more sensitive to authenticity in the analyses above, participants with more selective facial responses also showed larger authenticity discriminations between authentic and posed laughs in subjective judgments: the effect of the difference in average muscle activity between authentic and posed laughs over its observed range on the difference in authenticity ratings was 2.0 points for the zygomaticus $(95 \% \mathrm{CI}[0.9,3.1])$, and 1.8 for the orbicularis $(95 \% \mathrm{CI}[0.9,2.6])$.

\section{Electrodermal activity}

We observed SCRs, i.e., phasic changes in arousal, in $8.3 \%$ of the trials. The rate of these responses was higher for laughter compared to crying (odds ratio, $\mathrm{OR}=1.3,95 \% \mathrm{CI}$ $[1.0,1.7])$. Responses were also more frequent for authentic compared to posed laughs $(\mathrm{OR}=$ $1.5,95 \%$ CI $[1.1,2.0])$. For crying, there were no differences between authentic and posed stimuli $(\mathrm{OR}=0.9,95 \% \mathrm{CI}[0.6,1.2])$. As for the amplitude of SCRs, no differences were observed between authentic and posed vocalizations, for laughter $(\mathrm{OR}=1.2,95 \% \mathrm{CI}[0.9$, $1.4])$ and for crying $(\mathrm{OR}=1.1,95 \% \mathrm{CI}[0.9,1.3])$.

To examine whether electrodermal responses predicted authenticity evaluations, we first considered the frequency of SCRs. Authenticity ratings were not credibly higher in trials with and without SCRs (difference 0.1 points, 95\% CI [-0.04, 0.3]). There was a sizable, albeit statistically uncertain, tendency for male listeners to have SCRs in more trials compared to female listeners $(\mathrm{OR}=1.6,95 \% \mathrm{CI}[1.0,2.8])$. However, there was no pronounced interaction between SCRs and the sex or age of listeners (change in LOOIC from 
a model without demographics to models with an interaction with Sex or Age, respectively, was 3.2 with $S E=3.3$ for Sex and -3.2 with $S E=3.6$ for Age).

Regarding the amplitude of SCRs, there was only a small and statistically uncertain trend for higher authenticity ratings in trials with a more pronounced SCR. The highest vs. lowest observed SCR amplitude was predicted to produce authenticity ratings 0.4 points higher on a scale of 1 to $7(95 \%$ CI $[-0.7,1.5]$.

In short, phasic changes in arousal were more often evoked by authentic compared to posed laughs, but there was no association between this and authenticity judgments.

\subsection{Trait contagion and authenticity evaluations}

Average scores on the ECS were $3.7(S D=0.5)$, and on the IRI Empathic Concern subscale they were $2.8(S D=0.7)$. This is consistent with the published data for these measures (Davis, 1983; Doherty, 1997; Limpo et al., 2010; Rueff-Lopes \& Caetano, 2012). For the ECS, our results were in line with Neves et al.'s (2018). Higher trait contagion predicted higher authenticity ratings for authentic laughs (1.1 points higher for the highest vs. lowest contagion scores, $95 \%$ CI $[0.2,2.1]$, while it had no effect on posed laughs ( 0.1 points, $95 \%$ CI $[-1.1,1.3])$. For authenticity detection in crying, we found no associations with trait contagion (authentic cries, 0.3 points, 95\% CI [-0.7, 1.5]; posed cries, -0.8 points, $95 \%$ CI [$2.1,0.4])$. The effects of contagion on laughter authenticity ratings survived controlling for the listener's sex (1.07 higher ratings for authentic laughter for the highest vs. lowest contagion scores, 95\% CI [0.03, 2.2]; posed laughter, 0.1, 95\% [-1.19, 1.31]).

Regarding Empathic Concern scores, Neves et al.’s (2018) finding was only partially replicated. Higher scores predicted higher authenticity ratings in laughter (1.1 points higher for the highest vs. lowest empathic concern, 95\% CI [0.1, 2.1]). This does not translate into improved authenticity detection, however, because both authentic and posed laughs were evaluated as more authentic by participants with higher empathy scores (authentic laughs, 1.0 
points higher for the highest vs. lowest empathic concern, 95\% CI $[0.2,1.7]$; posed laughs, 1.1 points, $95 \% \mathrm{CI}[0.1,2.0])$. There was also no effect on authenticity ratings for crying (0.37 points higher for the highest vs. lowest empathic concern, 95\% CI $[-0.74,1.57])$, and exploratory analyses of the other IRI subscales have not shown a credible effect on authenticity detection in laughter or crying (effects on the authenticity ratings for laughter: perspective taking, $-0.03,95 \%$ CI [-0.77, 0.71]; fantasy, $0.11,95 \%$ CI [-0.63, 0.84]; personal distress, $-0.32,95 \%$ CI $[-1.10,0.46]$; effects on authenticity ratings for crying: perspective taking, $0.42,95 \%$ CI [-1.25, 0.44]; fantasy, $-0.38,95 \%$ CI [-1.17, 0.46]; personal distress; $0.08,95 \%$ CI $[-1.10,0.88])$.

In addition, we explored possible associations between ECS and IRI scores and physiological data, but no effects were found.

\section{Discussion}

The present study examined facial and electrodermal responses to laughter and crying. We investigated whether these responses are sensitive to the emotional authenticity of vocalizations, and whether they are associated with subsequent authenticity judgments. We present six main findings. First, facial responses were stronger for laughter compared to crying. In fact, responses to crying were not statistically different from baseline. Second, facial responses to laughter were observed in the zygomaticus and orbicularis, but not in the corrugator. Third, these responses were stronger for authentic compared to posed laughs, a result observed for average and peak levels of muscle activity. Fourth, facial activity was associated with authenticity ratings for laughter, but not for crying. For authentic and posed laughs, stronger responses in the orbicularis related to higher perceived authenticity. Stronger responses in the corrugator related to lower perceived authenticity. In the zygomaticus and orbicularis, participants showing more distinct responses to authentic and posed laughs also provided more distinct authenticity ratings in subjective evaluations. Fifth, laughter elicited 
phasic changes in electrodermal activity more often than crying, and authentic laughs elicited these responses more often than posed laughs. Electrodermal activity was not associated with authenticity evaluations, however. Sixth, participants reporting higher trait levels of emotional contagion showed improved authenticity detection in laughter.

Previous studies have shown that laughter is highly behaviorally contagious (Scott et al., 2014). Listening to laughter, more so than listening to negative vocalizations, recruits motor and premotor brain regions involved in the production of orofacial movements (Lima et al., 2015; McGettigan et al., 2015; O’Nions et al., 2017; Warren et al., 2006). In an fMRI study of passively listening to nonverbal vocalizations, for example, Warren et al. (2006) found a correlation between sensorimotor activity and valence. Activity in lateral premotor and primary motor cortices was greater in response to the positive sounds of laughter and cheers of triumph, and lower to the negative sounds of fear and disgust. Here, we provided evidence that the role of valence in sensorimotor responses to vocal emotions is also seen in peripheral facial responses. The absence of facial muscle activity in response to crying corroborates the relatively lower sensorimotor responses to negative sounds. The robust responses observed for laughter add to the contagiousness of this social expression. Moreover, responses to laughter were selectively seen on muscles that are associated with expressions of positive affect, the zygomaticus and the orbicularis (e.g., Dimberg et al., 2000; Dimberg et al., 2002; Korb et al., 2014). This suggests that the observed facial activity reflects a preparatory tendency to join in with others' positive emotions, consistent with the established role of laughter in the vicarious experience of positive affect, and in promoting affiliation and social connectedness (Gervais \& Wilson, 2005; Scott et al., 2014).

Another novel finding was that the emotional authenticity of laughter led to distinct responses in the zygomaticus and orbicularis. This is evidence that participants' facial responses differentiated authentic from posed laughs spontaneously, even in the absence of 
any task instructions related to authenticity. A similar differentiation has been reported in the visual domain, between Duchenne and non-Duchenne smiles (Krumhuber et al., 2014; Surakka \& Hietanen, 1998). Here we show that this effect can occur across sensory modalities, from heard vocalizations to facial responses. This finding is consistent with the notions that vocalizations and facial expressions are closely linked, and that there is crossmodal integration of the motor and affective systems involved in emotion processing (Arias et al., 2018; Hawk et al., 2012).

Regarding the literature on vocal emotional processing, previous evidence documents cortical sensitivities to laughter authenticity (McGettigan et al., 2015; O’Nions et al., 2017). The current study extends this sensitivity to peripheral nervous system responses. In fact, our results indicate that facial EMG might be particularly sensitive to fine-grained distinctions in underlying sensorimotor activity. Previous fMRI work failed to document differences between authentic and posed laughs in sensorimotor cortical systems, the main differences being in temporal and medial prefrontal and cingulate cortices (McGettigan et al., 2015; O’Nions et al., 2017). It might be that, for laughter, authenticity-related distinctions in sensorimotor activity are more easily captured in subtle facial responses than in the magnitude of sensorimotor cortex activity.

For crying, facial activity was similar for authentic and posed stimuli. Thus, not only were facial responses weaker to crying compared to laughter, but the authenticity distinction was also less clear. We cannot determine whether it is the case that the authenticity distinction for crying is less dependent on sensorimotor activity, or whether the lack of differences is a consequence of the lack of muscle activity for crying in general. Future studies will need to clarify this null result, because the lack of facial responses is no evidence for the absence of sensorimotor activity - sensorimotor activity might occur without resulting in facial responses (Wood et al., 2016). It will be interesting to compare cortical responses to 
authenticity in laughter and crying using more precise measurements such as multivariate methods or neural adaptation designs (e.g., Schmidt et al., 2020).

Consistent with the prediction that sensorimotor mechanisms play a role in emotion processing (e.g., Keysers \& Gazzola, 2009; Niedenthal et al., 2010; Wood et al., 2016), we found associations between facial muscles activity and the ratings of emotional authenticity of laughter. Orbicularis activity was related to perceptions of authentic affect, regardless of whether laughs were authentic or posed, and corrugator activity was related to perceptions of posed affect. That orbicularis activity related to perceptions of authentic affect is consistent with the stronger responses observed in this muscle for authentic laughs. It is also consistent with evidence from the facial literature that the orbicularis is involved in the perception and production of smiles, particularly when they are authentic (e.g., Hess \& Bourgeois, 2010; Korb et al., 2014; Krumhuber et al., 2014). Korb et al. (2014), for instance, found that orbicularis activity was higher in response to 'stronger' smiles, containing maximum levels of crow's feet (Duchenne's marker), and these were evaluated highest in authenticity.

That activity in the corrugator related to perceptions of posed affect might reflect the more negative valence often assigned to posed laughter. Authentic and posed laughs are typically assessed positively for valence, but valence ratings are often lower for posed laughs (Lavan et al., 2016; McGettigan et al., 2015). Considering the established association between the corrugator and negative affect (e.g., Dimberg, 1982; Dimberg et al., 2011; Wood et al., 2016), it is plausible that stronger activity in this muscle signaled higher negativity during laughter perception, which might have shaped perceptions of decreased authenticity. The corrugator has also been associated with cognitive control, however (Lindström et al., 2013; Schacht et al., 2008), so an alternative account could be that its activity signals the increased socio-emotional ambiguity of posed laughs rather than their negativity alone (McGettigan et al., 2015). 
Unexpectedly, we did not find a general association between zygomaticus activity and authenticity evaluations. Because the zygomaticus can be voluntarily contracted, more so than the orbicularis (Ekman, 2003), the signal might be noisier and therefore less reliably associated with behavior. However, we did find that participants showing more distinct responses in this muscle to authentic and posed laughs also showed a larger differentiation between the two types of laughs in authenticity evaluations. The same was observed for the orbicularis. From an individual differences perspective, this indicates that for the two muscles that are sensitive to laughter authenticity, the zygomaticus and orbicularis, more precise reactions as a function of laughter type can translate into more distinct authenticity evaluations.

The pattern of facial EMG and electrodermal results combined suggests that the association between facial activity and authenticity evaluations is not reducible to arousal. Authentic laughs are often higher in perceived arousal (e.g., Bryant et al., 2018; Lavan et al., 2016), so arousal could be a third variable causing stronger facial responses, higher authenticity ratings, and associations between the two. However, stronger facial responses were not always associated with higher authenticity ratings. There was a negative association between corrugator activity and perceived authenticity. Additionally, in the zygomaticus, higher muscle activity was not associated with higher perceived authenticity. Regarding electrodermal responses, we did find new evidence that authentic laughter more often triggers measurable phasic changes in arousal. Nevertheless, these responses were not linked to how participants evaluated laughter authenticity. It is therefore likely that the associations between facial responses and authenticity judgments is specific, plausibly reflecting the contribution of sensorimotor simulation, not an artifact of arousal.

A limitation of the current study is that we used a correlational design. We cannot exclude that facial EMG activity is the result, not a cause, of authenticity detection. 
Participants could have differentiated authentic from posed vocalizations first (e.g., based on auditory-perceptual analysis), and based on that showed distinct facial responses. We minimized this possibility by collecting physiological measurements before authenticity judgments, and while participants' attentional focus was unrelated to authenticity detection. However, some degree of automatic detection might still have occurred. We therefore document robust emotional authenticity effects on peripheral responding, but the causal direction of associations with behavior will require other approaches to be established with certainty. Experimental studies using mimicry suppression techniques will be useful to address this issue (e.g., Borgomaneri et al., 2020; Maringer et al., 2011; Oberman et al., 2007; Rychlowska et al., 2014). Another aspect is that we emphasized an authentic-posed distinction, but recent accounts have also emphasized laughter distinctions in terms of their social functions, namely reward, affiliation and dominance (Wood \& Niedenthal, 2018). It will be interesting to extend our approach to this categorization in future work.

We only partly succeeded in replicating our previous finding that the propensity to engage in sensorimotor activity is associated with authenticity detection (Neves et al., 2018). We had found that the ECS and IRI Empathic Concern scores correlated with improved authenticity detection. Here the effect was limited to the ECS. This could be because the effect is weak or due to task differences. While in Neves et al. (2018) the task was focused on laughter authenticity only, here the focus was on laughter and crying. Considering our findings that laughter elicits stronger facial responses, and more reliable associations with behavior, it could have been that the previous focus on laughter maximized the engagement of sensorimotor processes. Perceptual differences between authentic and posed laughs were also more subtle in our previous compared to the current study, and this was reflected in the magnitude of authenticity discrimination: 2.2 points in the current study compared to 1 point previously. The more challenging task used before could have contributed to maximizing 
sensorimotor activity too. Future work comparing task format effects in associations between the questionnaires and authenticity detection will be needed to clarify this issue.

To conclude, the present study is the first demonstration that emotional authenticity affects peripheral nervous system responses to nonverbal vocalizations, namely laughter, and that such responses relate to authenticity judgments. Compared to posed laughter, listening to authentic laughter elicited stronger facial responses in the zygomaticus and orbicularis, and more frequent arousal responses in electrodermal activity. Stronger responses in the orbicularis related to higher perceived laughter authenticity, and stronger responses in the corrugator related to lower perceived laughter authenticity. In the zygomaticus and orbicularis, participants showing more distinct responses to authentic and posed laughs also showed more distinct authenticity evaluations. Similar associations were not found for arousal responses, or for crying, which elicited weaker physiological responses in general. Altogether, these results are consistent with sensorimotor simulation models of emotion processing. They emphasize that the role of sensorimotor mechanisms is not limited to vision, potentially providing a route for emotion processing across modalities. 


\section{Acknowledgments}

During the preparation of this manuscript, César F. Lima was supported by a Fundação para a Ciência e Tecnologia (FCT) Investigator Grant (grant number IF/00172/2015). The preparation of auditory stimuli was funded by a Wellcome Trust Senior Research Fellowship (grant number WT090961MA) awarded to Sophie K. Scott. 


\section{References}

Adolphs, R., Damasio, H., Tranel, D., Cooper, G., \& Damasio, A. R. (2000). A role for somatosensory cortices in the visual recognition of emotion as revealed by threedimensional lesion mapping. Journal of Neuroscience, 20(7), 2683-2690. https://doi.org/10.1523/JNEUROSCI.20-07-02683.2000

Anikin, A., \& Lima, C. F. (2018). Perceptual and acoustic differences between authentic and acted nonverbal emotional vocalizations. Quarterly Journal of Experimental Psychology, 71(3), 622-641. https://doi.org/10.1080/17470218.2016.1270976

Argaud, S., Delplanque, S., Houvenaghel, J. F., Auffret, M., Duprez, J., Vérin, M., Grandjean, D. \& Sauleau, P. (2016). Does facial amimia impact the recognition of facial emotions? An EMG study in Parkinson's disease. PLOS One, 11(7), e0160329. https://doi.org/10.1371/journal.pone.0160329

Arias, P., Belin, P., \& Aucouturier, J-J. (2018). Auditory smiles trigger unconscious facial imitation. Current Biology, 28(14), R782-R783. https://doi.org/10.1016/j.cub.2018.05.084

Banissy, M. J., Sauter, D. A., Ward, J., Warren, J. E., Walsh, V., \& Scott, S. K. (2010). Suppressing sensorimotor activity modulates the discrimination of auditory emotions but not speaker identity. Journal of Neuroscience, 30(41), 13552-13557. https://doi.org/10.1523/JNEUROSCI.0786-10.2010

Bestelmeyer, P. E., Maurage, P., Rouger, J., Latinus, M., \& Belin, P. (2014). Adaptation to vocal expressions reveals multistep perception of auditory emotion. Journal of Neuroscience, 34(24), 8098-8105. https://doi.org/10.1523/JNEUROSCI.482013.2014 
Borgomaneri, S., Bolloni, C., Sessa, P., \& Avenanti, A. (2020). Blocking facial mimicry affects recognition of facial and body expressions. PLOS One, 15(2), e0229364. https://doi.org/10.1371/journal.pone.0229364

Braithwaite, J. J., Watson, D. G., Jones, R., \& Rowe, M. (2013). A guide for analysing electrodermal activity (EDA) \& skin conductance responses (SCRs) for psychological experiments. Psychophysiology, 49(1), 1017-1034.

http://www.biopac.com/manuals/EDA\%20SCR\%20Analysis.pdf

Bryant, G. A., \& Aktipis, C. A. (2014). The animal nature of spontaneous human laughter. Evolution and Human Behavior, 35(4), 327-335. https://doi.org/10.1016/j.evolhumbehav.2014.03.003

Bryant, G. A., Fessler, D. M. T., Fusaroli, R., Clint, E., Amir, D., Chávez, B., Denton, K. K., Díaz, C., Duran, L. T., Fanćovićová, J., Fux, M., Ginting, E. F., Hasan, Y., Hu, A., Kamble, S. V., Kameda, T., Kuroda, K., Li, N. P., Luberti, F. R., ... Zhou, Y. (2018). The perception of spontaneous and volitional laughter across 21 societies. Psychological Science, 29(9), 1515-1525. https://doi.org/10.1177/0956797618778235

Bürkner, P. C. (2017). brms: An R package for Bayesian multilevel models using Stan. Journal of Statistical Software, 80(1), 1-28. https://doi.org/10.18637/jss.v080.i01

Bürkner, P. C., \& Vuorre, M. (2019). Ordinal regression models in psychology: A tutorial. Advances in Methods and Practices in Psychological Science, 2(1), 77-101. https://doi.org/10.1177/2515245918823199

Correia, A. I., Branco, P., Martins, M., Reis, A. M., Martins, N., Castro, S. L., \& Lima, C. F. (2019). Resting-state connectivity reveals a role for sensorimotor systems in vocal 
emotional processing in children. NeuroImage, 201, 116052.

https://doi.org/10.1016/j.neuroimage.2019.116052

Cowen, A. S., Elfenbein, H. A., Laukka, P., \& Keltner, D. (2019). Mapping 24 emotions conveyed by brief human vocalization. American Psychologist, 74(6), 698. https://doi.org/10.1037/amp0000399

Davis, M. H. (1980). A multidimensional approach to individual differences in empathy. JSAS Catalog of Selected Documents in Psychology, 10, 85-103.

Davis, M. H. (1983). Measuring individual differences in empathy: Evidence for a multidimensional approach. Journal of Personality and Social Psychology, 44(1), 113. https://doi.org/10.1037/0022-3514.44.1.113

Dimberg, U. (1982). Facial reactions to facial expressions. Psychophysiology, 19(6), 643647. https://doi.org/10.1111/j.1469-8986.1982.tb02516.x

Dimberg, U., Andréasson, P., \& Thunberg, M. (2011). Emotional empathy and facial reactions to facial expressions. Journal of Psychophysiology, 25(1), 26. https://doi.org/10.1027/0269-8803/a000029

Dimberg, U., Thunberg, M., \& Elmehed, K. (2000). Unconscious facial reactions to emotional facial expressions. Psychological Science, 11(1), 86-89. https://doi.org/10.1111/1467-9280.00221

Dimberg, U., Thunberg, M., \& Grunedal, S. (2002). Facial reactions to emotional stimuli: Automatically controlled emotional responses. Cognition \& Emotion, 16(4), 449-471. https://doi.org/10.1080/02699930143000356 
Doherty, R. W. (1997). The emotional contagion scale: A measure of individual differences. Journal of Nonverbal Behavior, 21(2), 131-154. https://doi.org/10.1023/A:1024956003661

Ekman, P. (2003). Darwin, deception, and facial expression. Annals of the New York Academy of Sciences, 1000(1), 205-221. https://doi.org/10.1196/annals.1280.010

Fridlund, A. J., \& Cacioppo, J. T. (1986). Guidelines for human electromyographic research. Psychophysiology, 23(5), 567-589. https://doi.org/10.1111/j.14698986.1986.tb00676.x

Frühholz, S., Trost, W., \& Kotz, S. A. (2016). The sound of emotions - Towards a unifying neural network perspective of affective sound processing. Neuroscience $\&$ Biobehavioral Reviews, 68, 96-110. https://doi.org/10.1016/j.neubiorev.2016.05.002

Fujimura, T., Sato, W., \& Suzuki, N. (2010). Facial expression arousal level modulates facial mimicry. International Journal of Psychophysiology, 76(2), 88-92. https://doi.org/10.1016/j.ijpsycho.2010.02.008

Geangu, E., Quadrelli, E., Conte, S., Croci, E., \& Turati, C. (2016). Three-year-olds' rapid facial electromyographic responses to emotional facial expressions and body postures. Journal of Experimental Child Psychology, 144, 1-14. https://doi.org/10.1016/j.jecp.2015.11.001

Gervais, M., \& Wilson, D. S. (2005). The evolution and functions of laughter and humor: A synthetic approach. The Quarterly Review of Biology, 80(4), 395-430. https://doi.org/10.1086/498281 
Grainger, S. A., Vanman, E. J., Matters, G., \& Henry, J. D. (2019). The influence of tears on older and younger adults' perceptions of sadness. Psychology and Aging, 34(5), 665. https://doi.org/10.1037/pag0000373

Grandjean, D. (2020). Brain networks of emotional prosody processing. Emotion Review, 1754073919898522. https://doi.org/10.1177/1754073919898522

Hawk, S. T., Fischer, A. H., \& Van Kleef, G. A. (2012). Face the noise: Embodied responses to nonverbal vocalizations of discrete emotions. Journal of Personality and Social Psychology, 102(4), 796. https://doi.org/10.1037/a0026234

Hennenlotter, A., Schroeder, U., Erhard, P., Castrop, F., Haslinger, B., Stoecker, D., Lange, K. W., \& Ceballos-Baumann, A. O. (2005). A common neural basis for receptive and expressive communication of pleasant facial affect. NeuroImage, 26(2), 581-591. https:// doi.org/10.1016/j.neuroimage.2005.01.057

Hess, U., \& Blairy, S. (2001). Facial mimicry and emotional contagion to dynamic emotional facial expressions and their influence on decoding accuracy. International Journal of Psychophysiology, 40(2), 129-141. https://doi.org/10.1016/S0167-8760(00)00161-6

Hess, U., \& Bourgeois, P. (2010). You smile-I smile: Emotion expression in social interaction. Biological Psychology, 84(3), 514-520. https://doi.org/10.1016/j.biopsycho.2009.11.001

Hess, U. (2021). Who to whom and why: The social nature of emotional mimicry. Psychophysiology, 58, e13675. https://doi.org/10.1111/psyp.13675

Hietanen, J. K., Surakka, V., \& Linnankoski, I. (1998). Facial electromyographic responses to vocal affect expressions. Psychophysiology, 35(5), 530-536. https://doi.org/10.1017/S0048577298970445 
Hyniewska, S., \& Sato, W. (2015). Facial feedback affects valence judgments of dynamic and static emotional expressions. Frontiers in Psychology, 6, 291. https://doi.org/10.3389/fpsyg.2015.00291

Isomura, T., \& Nakano, T. (2016). Automatic facial mimicry in response to dynamic emotional stimuli in five-month-old infants. Proceedings of the Royal Society B: Biological Sciences, 283(1844), 20161948. https://doi.org/10.1098/rspb.2016.1948

Keysers, C., \& Gazzola, V. (2009). Expanding the mirror: Vicarious activity for actions, emotions, and sensations. Current Opinion in Neurobiology, 19(6), 666-671. https:// doi.org/10.1016/j.conb.2009.10.006

Korb, S., Grandjean, D., \& Scherer, K. R. (2010). Timing and voluntary suppression of facial mimicry to smiling faces in a Go/NoGo task - An EMG study. Biological Psychology, 85(2), 347-349. https://doi.org/10.1016/j.biopsycho.2010.07.012

Korb, S., Malsert, J., Rochas, V., Rihs, T. A., Rieger, S. W., Schwab, S., Niedenthal, P. M., \& Grandjean, D. (2015). Gender differences in the neural network of facial mimicry of smiles-An rTMS study. Cortex, 70, 101-114. https://doi.org/10.1016/j.cortex.2015.06.025

Korb, S., Niedenthal, P., Kaiser, S., \& Grandjean, D. (2014). The perception and mimicry of facial movements predict judgments of smile authenticity. PLOS One, 9(6), e99194. https://doi.org/10.1371/journal.pone.0099194

Krumhuber, E. G., Likowski, K. U., \& Weyers, P. (2014). Facial mimicry of spontaneous and deliberate Duchenne and non-Duchenne smiles. Journal of Nonverbal Behavior, 38(1), 1-11. https://doi.org/10.1007/s10919-013-0167-8 
Künecke, J., Hildebrandt, A., Recio, G., Sommer, W., \& Wilhelm, O. (2014). Facial EMG responses to emotional expressions are related to emotion perception ability. PLOS One, 9(1), e84053. https://doi.org/10.1371/journal.pone.0084053

Lapatki, B. G., Stegeman, D. F., \& Jonas, I. E. (2003). A surface EMG electrode for the simultaneous observation of multiple facial muscles. Journal of Neuroscience Methods, 123(2), 117-128. https://doi.org/10.1016/S0165-0270(02)00323-0

Lavan, N., Lima, C. F., Harvey, H., Scott, S. K., \& McGettigan, C. (2015). I thought that I heard you laughing: Contextual facial expressions modulate the perception of authentic laughter and crying. Cognition and Emotion, 29(5), 935-944. https://doi.org/10.1080/02699931.2014.957656

Lavan, N., Scott, S. K., \& McGettigan, C. (2016). Laugh like you mean it: Authenticity modulates acoustic, physiological and perceptual properties of laughter. Journal of Nonverbal Behavior, 40(2), 133-149. https://doi.org/10.1007/s10919-015-0222-8

Lima, C. F., Alves, T., Scott, S. K., \& Castro, S. L. (2014). In the ear of the beholder: How age shapes emotion processing in nonverbal vocalizations. Emotion, 14(1), 145. https://doi.org/10.1037/a0034287

Lima, C. F., Anikin, A., Monteiro, A. C., Scott, S. K., \& Castro, S. L. (2019). Automaticity in the recognition of nonverbal emotional vocalizations. Emotion, 19(2), 219-233. https://doi.org/10.1037/emo0000429

Lima, C. F., Brancatisano, O., Fancourt, A., Müllensiefen, D., Scott, S. K., Warren, J. D., \& Stewart, L. (2016a). Impaired socio-emotional processing in a developmental music disorder. Scientific Reports, 6, 34911. https://doi.org/10.1038/srep34911 
Lima, C. F., Castro, S. L., \& Scott, S. K. (2013). When voices get emotional: A corpus of nonverbal vocalizations for research on emotion processing. Behavior Research Methods, 45(4), 1234-1245. https://doi.org/10.3758/s13428-013-0324-3

Lima, C. F., Krishnan, S., \& Scott, S. K. (2016b). Roles of supplementary motor areas in auditory processing and auditory imagery. Trends in Neurosciences, 39(8), 527-542. https://doi.org/10.1016/j.tins.2016.06.003

Lima, C. F., Lavan, N., Evans, S., Agnew, Z., Halpern, A. R., Shanmugalingam, P., Meekings, S., Boebinger, D., Ostarek, M., McGettigan, C., Warren, J. E., \& Scott, S. K. (2015). Feel the noise: Relating individual differences in auditory imagery to the structure and function of sensorimotor systems. Cerebral Cortex, 25(11), 4638-4650. https://doi.org/10.1093/cercor/bhv134

Limpo, T., Alves, R. A., \& Castro, S. L. (2010). Medir a empatia: Adaptação portuguesa do índice de reactividade interpessoal [Measuring empathy: Portuguese adaptation of the interpersonal reactivity index]. Laboratório de Psicologia, 8, 171-184. https://doi.org/10.14417/lp.640

Lindström, B., Mattsson-Marn, Golkar, A., \& Olsson (2013). In your face: Risk of punishment enhanced cognitive control and error-related activity in the corrugator supercilii muscle. PLOS One, 8(6). e65692. https://doi.org/10.1371/journal.pone.0065692

Lobmaier, J. S., \& Fischer, M. H. (2015). Facial feedback affects perceived intensity but not quality of emotional expressions. Brain Sciences, 5(3), 357-368. https://doi.org/10.3390/brainsci5030357 
Magnée, M. J., Stekelenburg, J. J., Kemner, C., \& de Gelder, B. (2007). Similar facial electromyographic responses to faces, voices, and body expressions. Neuroreport, 18(4), 369-372. https://doi.org/10.1097/WNR.0b013e32801776e6

Maringer, M., Krumhuber, E. G., Fischer, A. H., \& Niedenthal, P. M. (2011). Beyond smile dynamics: Mimicry and beliefs in judgments of smiles. Emotion, 11(1), 181. https://doi.org/10.1037/a0022596

McGettigan, C., Walsh, E., Jessop, R., Agnew, Z. K., Sauter, D. A., Warren, J. E., \& Scott, S. K. (2015). Individual differences in laughter perception reveal roles for mentalizing and sensorimotor systems in the evaluation of emotional authenticity. Cerebral Cortex, 25(1), 246-257. https://doi.org/10.1093/cercor/bht227

McKeown, G., Sneddon, I., \& Curran, W. (2015). Gender differences in the perceptions of authentic and simulated laughter and amused facial expressions. Emotion Review, 7(1), 30-38. https://doi.org/10.1177/1754073914544475

Neves, L., Cordeiro, C., Scott, S. K., Castro, S. L., \& Lima, C. F. (2018). High emotional contagion and empathy are associated with enhanced detection of emotional authenticity in laughter. Quarterly Journal of Experimental Psychology, 71(11), 23552363. https://doi.org/10.1177/1747021817741800

Niedenthal, P. M., Mermillod, M., Maringer, M., \& Hess, U. (2010). The Simulation of Smiles (SIMS) model: Embodied simulation and the meaning of facial expression. Behavioral and Brain Sciences, 33(6), 417-433. https://doi.org/10.1017/S0140525X10000865 
Oberman, L. M., Winkielman, P., \& Ramachandran, V. S. (2007). Face to face: Blocking facial mimicry can selectively impair recognition of emotional expressions. Social Neuroscience, 2(3-4), 167-178. https://doi.org/10.1080/17470910701391943

O’Nions, E., Lima, C. F., Scott, S. K., Roberts, R., McCrory, E. J., \& Viding, E. (2017). Reduced laughter contagion in boys at risk for psychopathy. Current Biology, 27(19), 3049-3055. https://doi.org/10.1016/j.cub.2017.08.062

Oliva, M., \& Anikin, A. (2018). Pupil dilation reflects the time course of emotion recognition in human vocalizations. Scientific Reports, 8, 4871. https://doi.org/10.1038/s41598$018-23265-\mathrm{x}$

Pourtois, G., Sander, D., Andres, M., Grandjean, D., Reveret, L., Olivier, E., \& Vuilleumier, P. (2004). Dissociable roles of the human somatosensory and superior temporal cortices for processing social face signals. European Journal of Neuroscience, 20(12), 3507-3515. https://doi.org/10.1111/j.1460-9568.2004.03794.x

Rueff-Lopes, R., \& Caetano, A. (2012). The emotional contagion scale: Factor structure and psychometric properties in a Portuguese sample. Psychological Reports, 111(3), 898904. https://doi.org/10.2466/08.21.28.PR0.111.6.898-904

Rychlowska, M., Cañadas, E., Wood, A., Krumhuber, E. G., Fischer, A., \& Niedenthal, P. M. (2014). Blocking mimicry makes true and false smiles look the same. PLOS One, 9(3), e90876. https://doi.org/10.1371/journal.pone.0090876

Sauter, D. A., \& Eimer, M. (2010). Rapid detection of emotion from human vocalizations. Journal of Cognitive Neuroscience, 22(3), 474-481. https://doi.org/10.1162/jocn.2009.21215 
Sauter, D. A., Eisner, F., Calder, A. J., \& Scott, S. K. (2010a). Perceptual cues in nonverbal vocal expressions of emotion. Quarterly Journal of Experimental Psychology, 63(11), 2251-2272. https://doi.org/10.1080/17470211003721642

Sauter, D. A., Eisner, F., Ekman, P., \& Scott, S. K. (2010b). Cross-cultural recognition of basic emotions through nonverbal emotional vocalizations. Proceedings of the National Academy of Sciences, 107(6), 2408-2412. https://doi.org/10.1073/pnas.0908239106

Schacht, A., Nigbur, R., \& Sommer, W. (2008). Emotions in Go/NoGo conflicts. Psychological Research, 73, 843-856. https://doi.org/10.1007/s00426-008-0192-0

Schirmer, A., \& Kotz, S. A. (2006). Beyond the right hemisphere: Brain mechanisms mediating vocal emotional processing. Trends in Cognitive Sciences, 10(1), 24-30. https://doi.org/10.1016/j.tics.2005.11.009

Schmidt, S. N., Sojer, C. A., Hass, J., Kirsch, P., \& Mier, D. (2020). fMRI adaptation reveals: The human mirror neuron system discriminates emotional valence. Cortex. https://doi.org/10.1016/j.cortex.2020.03.026

Scott, S. K., Lavan, N., Chen, S., \& McGettigan, C. (2014). The social life of laughter. Trends in Cognitive Sciences, 18(12), 618-620. https://doi.org/10.1016/j.tics.2014.09.002

Surakka, V., \& Hietanen, J. K. (1998). Facial and emotional reactions to Duchenne and nonDuchenne smiles. International Journal of Psychophysiology, 29(1), 23-33. https://doi.org/10.1016/S0167-8760(97)00088-3

Warren, J. E., Sauter, D. A., Eisner, F., Wiland, J., Dresner, M. A., Wise, R. J., Rosen, S., \& Scott, S. K. (2006). Positive emotions preferentially engage an auditory-motor 
“mirror” system. Journal of Neuroscience, 26(50), 13067-13075.

https://doi.org/10.1523/JNEUROSCI.3907-06.2006

Wood, A., \& Niedenthal, P. (2018). Developing a social functional account of laughter. Social and Personality Psychology Compass, 12, e12383.

https://doi.org/10.1111/spc3.12383

Wood, A., Rychlowska, M., Korb, S., \& Niedenthal, P. (2016). Fashioning the face: Sensorimotor simulation contributes to facial expression recognition. Trends in Cognitive Sciences, 20(3), 227-240. https://doi.org/10.1016/j.tics.2015.12.010 


\section{Author Notes}

Funded by a grant from Portuguese Foundation for Science and Technology (FCT) awarded to C.F.L. (IF/ 00172/2015).

The preparation of auditory stimuli was funded by a Wellcome Trust Senior Research Fellowship (grant number WT090961MA) awarded to Sophie K. Scott. 


\section{Figure Captions}

Figure 1. Illustration of the experimental setup (A) and of the effects of muscle activity on facial features (B). Used with permission from Camilla Silveira and César Lima.

Figure 2. Authenticity ratings of authentic and posed laughter and crying. The points show fitted values from ordinal mixed models, with 95\% CIs. The violin plots show the distribution of observed ratings aggregated per sound. The dashed line indicates the neutral mid-point of the scale.

Figure 3. Facial activity in the orbicularis, zygomaticus and corrugator muscles during passive listening to authentic and posed laughter and crying. The graphs show percent change as compared to pre-trial baseline, as a time series smoothed with cubic regression splines (A), or as average and peak change considering all time bins after sound onset (B).

Figure 4. The effect of average facial muscles activity on authenticity ratings. Shown: the predicted effect of increasing average facial responses over their observed range of values. 


\section{Figures}

Figure 1
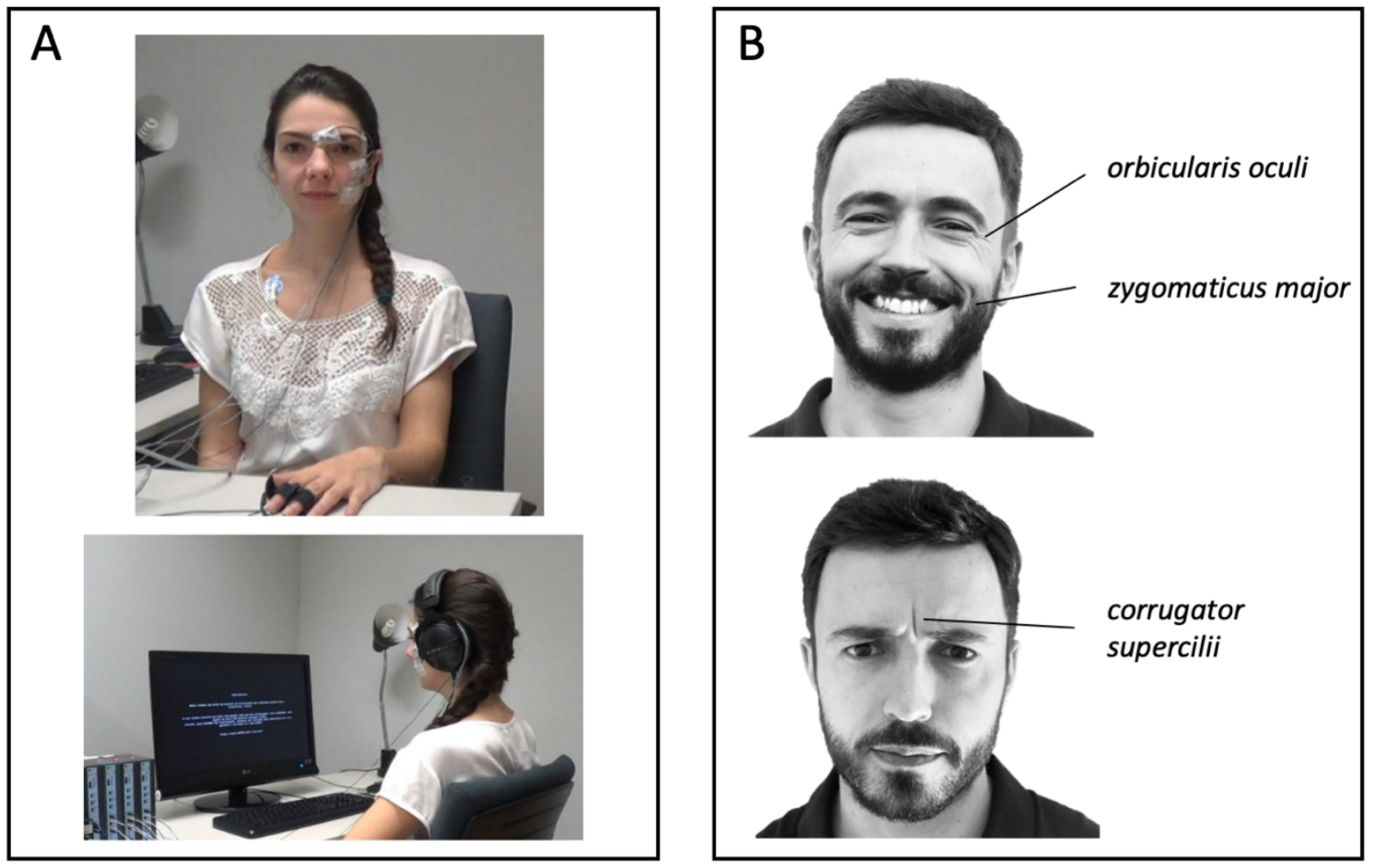
Figure 2

4 Posed $\phi$ Authentic

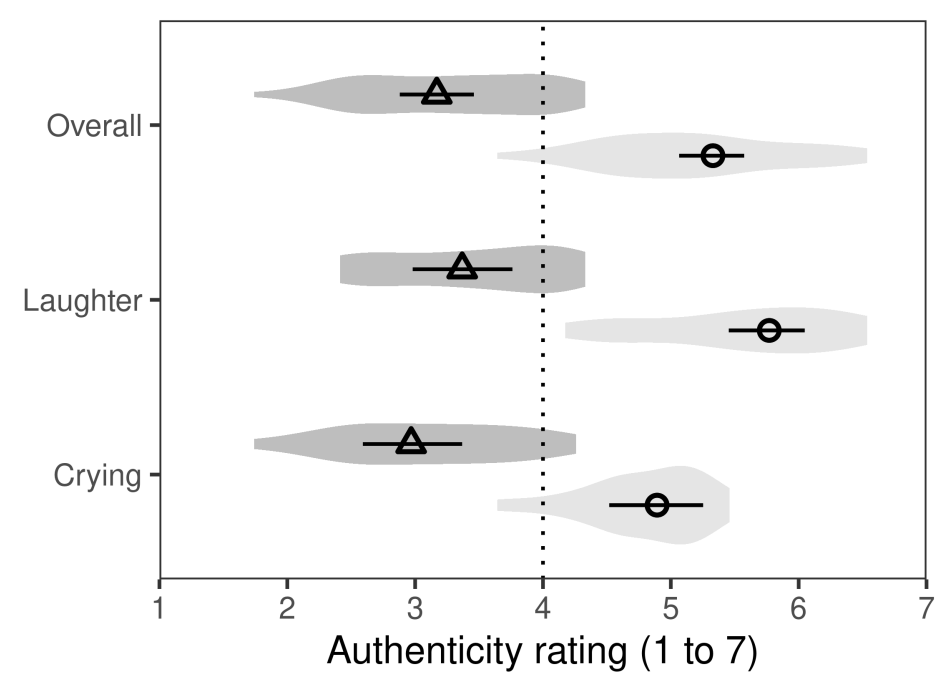


Figure 3

A

Orbicularis

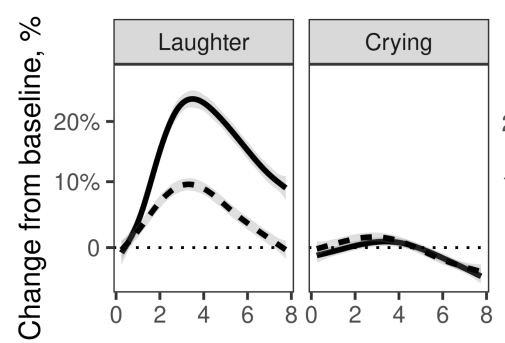

Zygomaticus

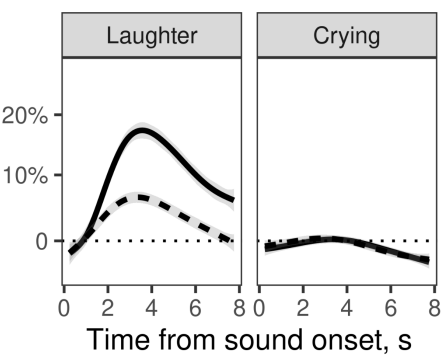

Corrugator
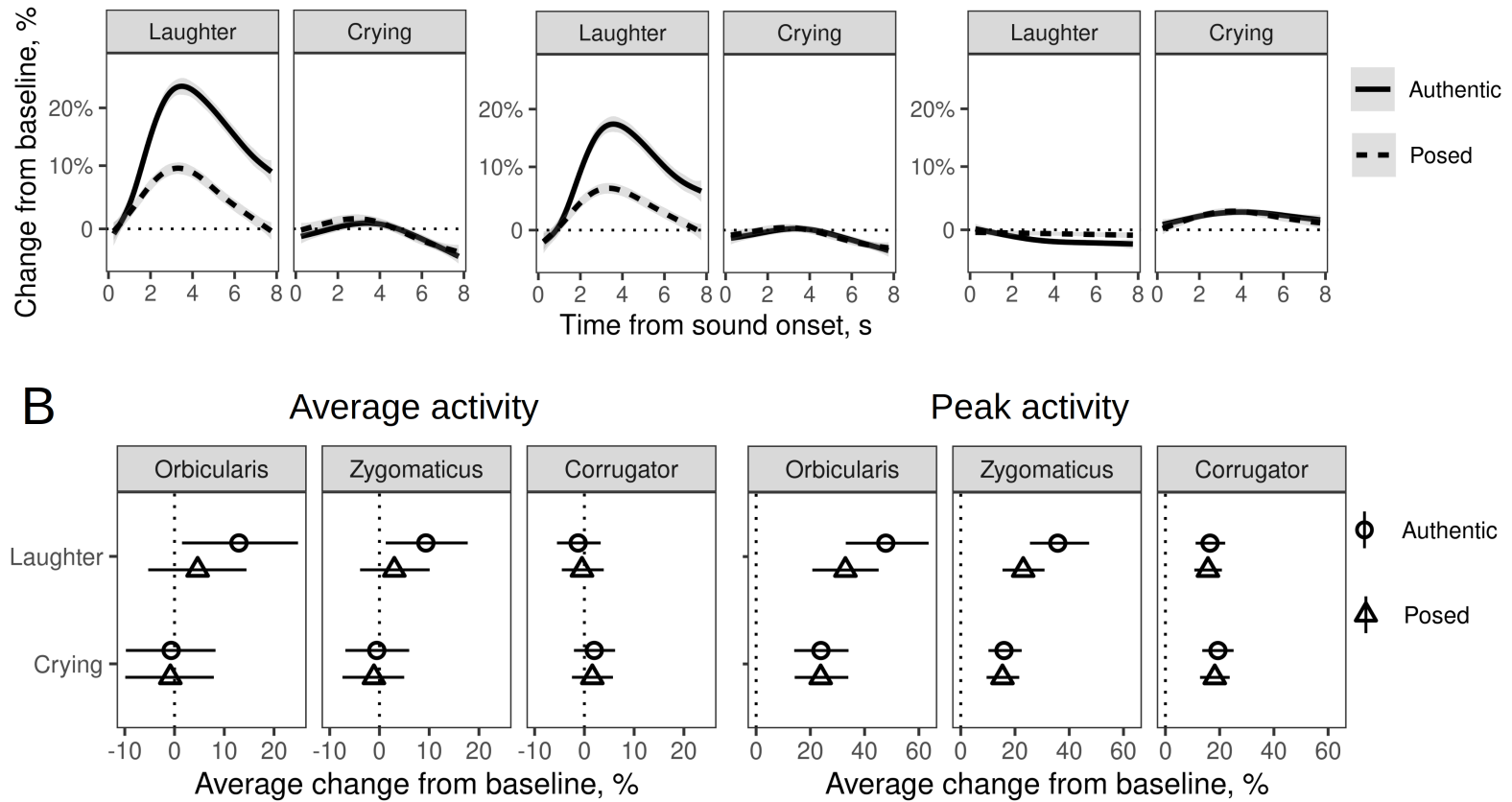

Peak activity

Average activity
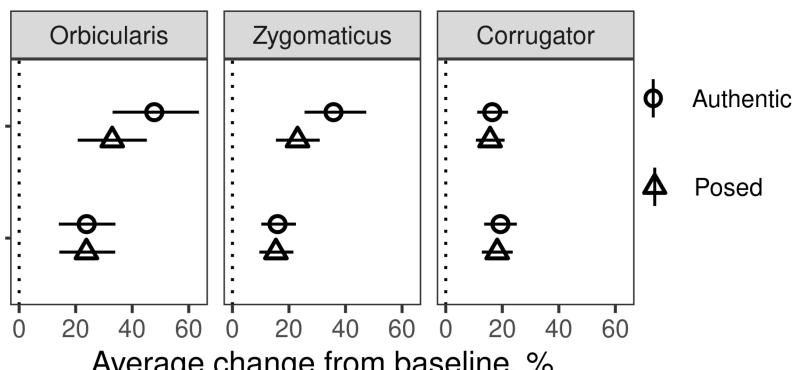
Figure 4

$$
\phi \text { Authentic } 4 \text { Posed }
$$

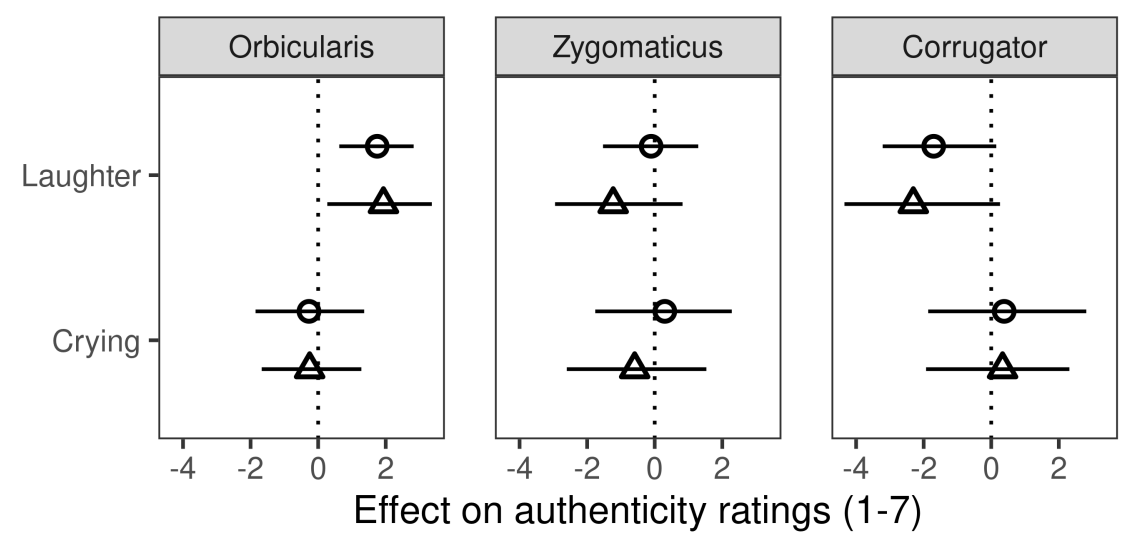

